\title{
Using neural networks to bridge scales in cancer: Mapping signaling pathways to phenotypes
}

\author{
Eunjung Kim- PSOC Member, Philip Gerlee, Alexander R.A. Anderson -PSOC Member
}

Cancer is an evolving system subject to mutation and selection. Selection is driven by the microenvironment that the cancer cells are growing in and acts on the cell phenotype, which is in turn modulated by intracellular signaling pathways regulated by the cell genotype. Integrating all of these processes requires bridging different biological scales. We present a mathematical model that uses a neural network as a means to connecting these scales. In particular, we consider the mapping from intracellular pathway activity to phenotype under different microenvironmental conditions.

\section{BRidging SCALES IN CANCER: From Pathway to PHENOTYPE}

Much effort has been made to define molecular characteristics of cancer progression. This has led to the development of targeted therapies that successfully control cancer initially. The success, however, is generally short lived as drug resistance eventually emerges (1), primarily due to the multiscale aspects of cancer (2) and heterogeneity (3). Understanding how the resistance develops and can be delayed is one of major focus of cancer research (4). A strategy to deal with this resistance is to consider combination therapies with existing drugs. To develop more effective strategies, it is required to understand how selection by drugs affects intracellular pathway signaling and how the altered signaling affects the cell phenotype under dynamic and heterogeneous microenvironments.

Understanding the relationship between different intracellular molecules and relating their interactions to a cell phenotype is an overwhelm task considering the vast number of simultaneous interactions occurring within a cancer cell. Mathematical modeling may be a way forward with its ability to integrate these dynamics and to describe both linear and non-linear feedback between interacting molecules (5). A number of previous studies have used mathematical models for describing intra-cellular dynamics, utilizing a spectrum of techniques including Boolean, logic, artificial neural networks, and ordinary differential equations (6-26). Here, we focus on a neural network modeling approach as a means to connecting intracellular pathway and phenotype scale under different microenvironmental conditions.

Research supported by Moffitt Cancer Center Physical Science Oncology Center, NIH/NCI U54CA193489.

Eunjung Kim is in Integrated Mathematical Oncology, H. Lee Moffitt Cancer and Research Institute, Tampa, FL (Eunjung.Kim@ moffitt.org).

Philip Gerlee is at Mathematical Sciences, Chalmers University of Technology, Gothenburg, Sweden (gerlee@chalmers.se).

Alexander R.A. Anderson is in Integrated Mathematical Oncology, H. Lee Moffitt Cancer and Research Institute, Tampa, FL, 33612, USA, (Alexander.Anderson@moffitt.org).

\section{Neural network Model in CANCER Cell Signaling}

We utilized a neural network modeling approach to determine cancer cell phenotypes affected by both the microenvironment and intracellular pathway activity (Fig. 1). Feeding the microenvironmental variables to the hidden intracellular pathway layer determines the output of the network, cell phenotype (Fig. 1). In order to capture the fact that proteins in signaling pathway regulate other proteins, we allow for regulatory feedback between proteins. This modeling approach and preliminary results have already been published (12).

We model the mitogen-activated protein kinase (MAPK) pathway and the PI3K/AKT pathway (Fig. 1) since the pathways are known to mainly regulate cancer cell growth and death (27). Using the model, we first study normal signaling responses in different microenvironments, various concentrations of growth factors and death promoting signal. The normal cell network produces a profile of the proteins in Figure 2 in four different microenvironmental conditions, (i) low growth but high death, (ii) high growth and high death, (iii) high growth and low death, and (iv) low growth and low death. The predicted pro-growth outputs reflect different contexts. For example, the value is low in the micrioenvironment (i) and increases in growth favoring microenvironments (e.g., (iii)). The microenvironment (iv) pushes cells to an inactive state by decreasing all protein levels (Fig. 2).

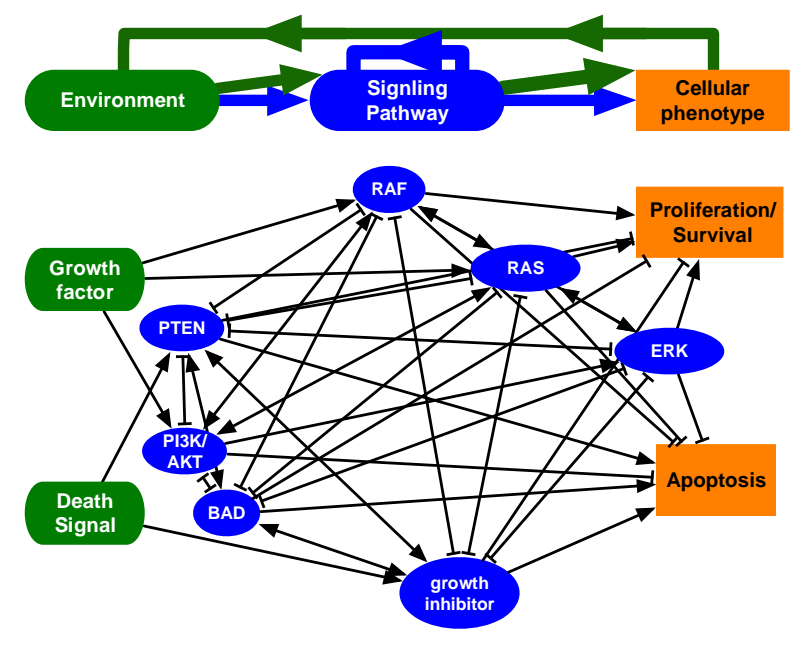

Figure 1. Artificial neural network. Microenvironmental variables (green) are in the input-layer and fed through the pathway (blue) and phenotype (orange), output layer. Connections between layers represent mapping from microenvironments to pathway and then pathway to phenotype. 
A

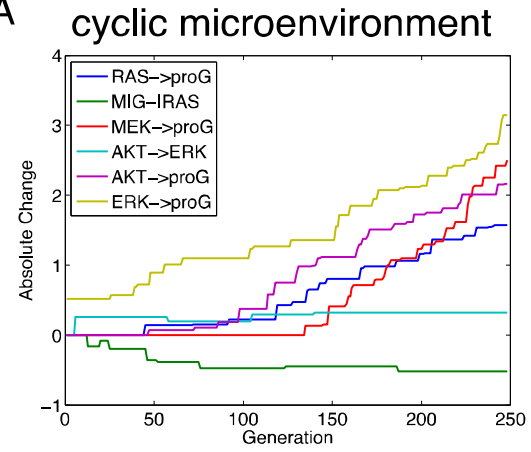

B

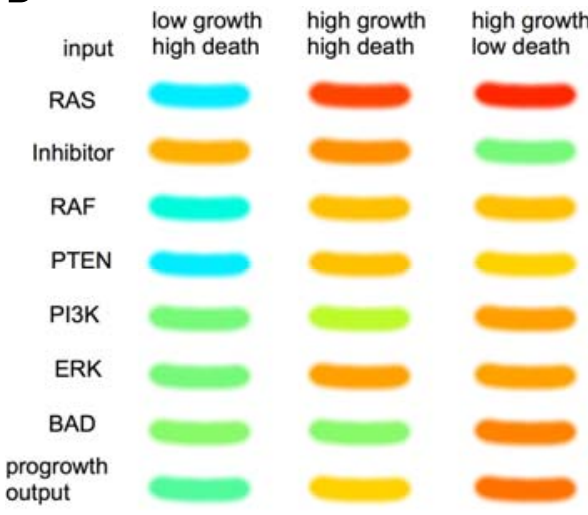

low growth factor

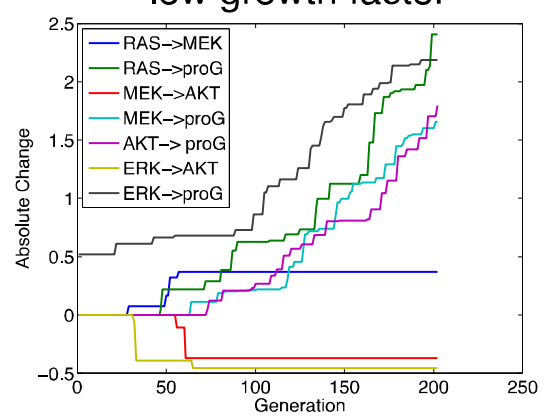

C
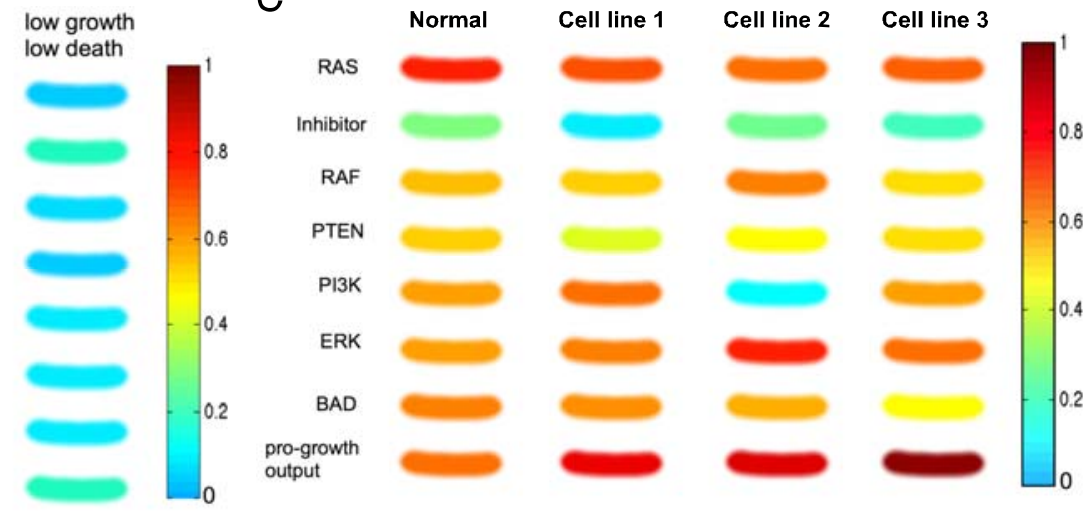

Figure 2. A: Changes of Weights from internal proteins to pro-growth output (proG) in a representative in silico cancer cell from cyclic, low, high growth factor microenvironment over 200 generations. B: Predicted protein activity and pro-growth output of normal cell in four different microenvironments indicated by the label on the top. C: Predicted protein activity as well as pro-growth output of normal and cancerous cell line (1-3) in microenvironment condition (iii), the high growth factor and low death factor condition.

Next, we evolve the normal network under different microenvironmental constraints to derive a cancer-signaling network. The network is evolved in three different microenvironments ((i) cyclic, (ii) low growth/high death, (iii) high growth/low death) to generate three different in silico cancer cell lines (cell line 1- cell line 3). We compare evolved weights of a representative in silico cancer cell from each microenvironment (Figure 2). The weights between internal proteins and pro-growth output seem to be increasing. We then compare the typical protein level of the normal network and three evolved cell lines (Fig. 2) in the high growth factor \& low-death factor microenvironment (iii). Cell line 1 has a significantly lower than normal expression of the inhibitor protein (cyan) which suggests that it may harbor an inactivating mutation. Cell line 2 may have activating mutations in RAF and ERK as well as an inactivating mutation in PI3K. The expression of cell line 3 is only slightly different from that of a normal cell. Since cell line 3 was evolved in the grow-promoting environment, only a slight change in the network was sufficient to satisfy the given criteria (i.e., high pro-growth output).

\section{Quick Guide to the Methods}

The network consists of two microenvironmental input nodes (growth factor and death signal), and two phenotype output nodes (pro-growth and pro-death), and several nodes that represent intra-cellular proteins. Only the internal nodes contain recurrent interactions. The rates of change of both protein level and phenotypes are determined as an additive linear combination of its neighbors weighted by interaction strength $(\mathbf{W})$, which is described by

$$
\frac{d y_{i}}{d t}=T\left(\sum_{j \in N(i)} W_{i j} y_{j}\right)-\lambda y_{i}, y_{i}(0)=0
$$

where $\lambda$ is a decay rate. The transfer function $T$ was considered $\left(T(z) \equiv \frac{1}{1+e^{-\beta z}}\right)$ to account for saturation effect. Of note, $y_{1}$ and $y_{2}$ are external pro-growth and pro-death inputs.

To obtain a weight matrix, we use Monte Carlo simulation. A weight matrix is initialized with random numbers. With the initialized weight, we solve equation (1). Then we randomly select a weight element and perturb it. With this perturbed weight, equation (1) is solved again. The steady state solution with the perturbed weight and the one with the unperturbed weight are used to evaluate a pre-defined cost function. If the cost is closer smaller, the perturbation is accepted. Otherwise, the perturbation is discarded. The process is iterated until convergence.

\section{A. Derivation of normal cell weight matrix}

In a normal cell, we assumed that the phenotype of the cell is directly regulated by microenvironmental cues. In 
other words, for a normal cell in a growth promoting microenvironment, it is more likely to reproduce. In a growth inhibiting or death promoting environment, a cell is less likely to divide and will have a higher chance of cell death. We model these phenomena using a cost function $C=\left|y_{1}-y_{g}\right|+\left|y_{2}-y_{d}\right|$, where $y_{1}$ and $y_{2}$ are given progrowth and pro-death inputs, respectively and $y_{g}$ and $y_{d}$ are pro-growth and pro-death outputs, respectively.

\section{B. Derivation of cancer cell weight matrix}

A mutation is modeled by randomly perturbing each element of the normal cell weight matrix. To model higher growth rate of a cancer cell, a new cost function $C=1-y_{g}$ was employed. Cell line 1 is evolved in condition (i), cell line 2 in (ii), and cell line 3 is evolved in the condition (iii).

\section{OTHER APPLICATIONS}

Artificial neural networks have been used as a machine learning approach for the detection of heart abnormalities (28-30) and cancer prediction (31-41). Our approach was also previously used to investigate the impact of the microenvironment on cancer growth and evolution (42-46), where the neural networks were embedded into an individual cell based model, hybrid cellular automata model, which allows for mutation in the cancer cells and subsequent selection by the microenvironment. A recent study utilizing similar approach highlighted the impact of targeted therapy on cell signaling heterogeneity (47).

\section{REFERENCES}

1. Ellis LM, Hicklin DJ. Resistance to Targeted Therapies: Refining Anticancer Therapy in the Era of Molecular Oncology. Clin Cancer Res. 2009;15(24):7471-8.

2. Anderson AR, Quaranta V. Integrative mathematical oncology. Nat Rev Cancer. 2008;8(3):227-34

3. Gerlinger M, Rowan AJ, Horswell S, Math M, Larkin J, Endesfelder D, et al. Intratumor heterogeneity and branched evolution revealed by multiregion sequencing. N Engl J Med. 2012;366(10):883-92.

4. Holohan C, Van Schaeybroeck S, Longley DB, Johnston PG. Cancer drug resistance: an evolving paradigm. Nat Rev Cancer. 2013;13(10):71426.

5. Byrne HM. Dissecting cancer through mathematics: from the cell to the animal model. Nat Rev Cancer. 2010;10(3):221-30.

6. Aldridge BB, Burke JM, Lauffenburger DA, Sorger PK. Physicochemical modelling of cell signalling pathways. Nat Cell Biol. 2006;8(11):1195-203.

7. Aldridge BB, Saez-Rodriguez J, Muhlich JL, Sorger PK, Lauffenburger DA. Fuzzy logic analysis of kinase pathway crosstalk in TNF/EGF/insulininduced signaling. PLoS Comput Biol. 2009;5(4):e1000340.

8. Cho CR, Labow M, Reinhardt M, van Oostrum J, Peitsch MC. The application of systems biology to drug discovery. Curr Opin Chem Biol. 2006;10(4):294-302.

9. Christopher R, Dhiman A, Fox J, Gendelman R, Haberitcher T, Kagle D, et al. Data-driven computer simulation of human cancer cell. Ann N Y Acad Sci. 2004;1020:132-53.

10.di Bernardo D, Thompson MJ, Gardner TS, Chobot SE, Eastwood EL, Wojtovich AP, et al. Chemogenomic profiling on a genome-wide scale using reverse-engineered gene networks. Nat Biotechnol. 2005;23(3):37783 .

11.Gardner TS, di Bernardo D, Lorenz D, Collins JJ. Inferring genetic networks and identifying compound mode of action via expression profiling. Science. 2003;301(5629):102-5.
12.Gerlee P, Kim E, Anderson AR. Bridging scales in cancer progression: mapping genotype to phenotype using neural networks. Semin Cancer Biol. 2015;30:30-41.

13.Kabir M, Noman N, Iba H. Reverse engineering gene regulatory network from microarray data using linear time-variant model. BMC Bioinformatics. 2010;11 Suppl 1:S56.

14.Korkut A, Wang W, Demir E, Aksoy BA, Jing X, Molinelli EJ, et al. Perturbation biology nominates upstream-downstream drug combinations in RAF inhibitor resistant melanoma cells. Elife. 2015;4.

15.Kumar N, Hendriks BS, Janes KA, de Graaf D, Lauffenburger DA. Applying computational modeling to drug discovery and development. Drug Discov Today. 2006;11(17-18):806-11.

16.Le Novere N. Quantitative and logic modelling of molecular and gene networks. Nat Rev Genet. 2015;16(3):146-58.

17.Luan D, Zai M, Varner JD. Computationally derived points of fragility of a human cascade are consistent with current therapeutic strategies. PLoS Comput Biol. 2007;3(7):e142.

18.Molinelli EJ, Korkut A, Wang W, Miller ML, Gauthier NP, Jing X, et al. Perturbation biology: inferring signaling networks in cellular systems. PLoS Comput Biol. 2013;9(12):e1003290.

19.Morris MK, Saez-Rodriguez J, Clarke DC, Sorger PK, Lauffenburger DA. Training signaling pathway maps to biochemical data with constrained fuzzy logic: quantitative analysis of liver cell responses to inflammatory stimuli. PLoS Comput Biol. 2011;7(3):e1001099.

20.Nakakuki T, Birtwistle MR, Saeki Y, Yumoto N, Ide K, Nagashima T, et al. Ligand-specific c-Fos expression emerges from the spatiotemporal control of ErbB network dynamics. Cell. 2010;141(5):884-96.

21.Saez-Rodriguez J, Alexopoulos LG, Epperlein J, Samaga R, Lauffenburger DA, Klamt S, et al. Discrete logic modelling as a means to link protein signalling networks with functional analysis of mammalian signal transduction. Mol Syst Biol. 2009;5:331.

22.Samaga R, Saez-Rodriguez J, Alexopoulos LG, Sorger PK, Klamt S. The logic of EGFR/ErbB signaling: theoretical properties and analysis of high-throughput data. PLoS Comput Biol. 2009;5(8):e1000438.

23.Schoeberl B, Eichler-Jonsson C, Gilles ED, Muller G. Computational modeling of the dynamics of the MAP kinase cascade activated by surface and internalized EGF receptors. Nat Biotechnol. 2002;20(4):370-5.

24.Steffen M, Petti A, Aach J, D'Haeseleer P, Church G. Automated modelling of signal transduction networks. BMC Bioinformatics. 2002;3:34.

25.Wang DY, Cardelli L, Phillips A, Piterman N, Fisher J. Computational modeling of the EGFR network elucidates control mechanisms regulating signal dynamics. BMC Syst Biol. 2009;3:118.

26.Wynn ML, Consul N, Merajver SD, Schnell S. Logic-based models in systems biology: a predictive and parameter-free network analysis method. Integr Biol (Camb). 2012;4(11):1323-37.

27.Smalley KS. Understanding melanoma signaling networks as the basis for molecular targeted therapy. J Invest Dermatol. 2010;130(1):28-37.

28.Baxt WG. Use of an artificial neural network for the diagnosis of myocardial infarction. Ann Intern Med. 1991;115(11):843-8.

29.Eggers KM, Ellenius J, Dellborg M, Groth T, Oldgren J, Swahn E, et al. Artificial neural network algorithms for early diagnosis of acute myocardial infarction and prediction of infarct size in chest pain patients. Int J Cardiol. 2007;114(3):366-74.

30.Kennedy RL, Harrison RF, Burton AM, Fraser HS, Hamer WG, MacArthur D, et al. An artificial neural network system for diagnosis of acute myocardial infarction (AMI) in the accident and emergency department: evaluation and comparison with serum myoglobin measurements. Comput Methods Programs Biomed. 1997;52(2):93-103.

31.Abdolmaleki P, Buadu LD, Naderimansh H. Feature extraction and classification of breast cancer on dynamic magnetic resonance imaging using artificial neural network. Cancer Lett. 2001;171(2):183-91.

32.Bassi P, Sacco E, De Marco V, Aragona M, Volpe A. Prognostic accuracy of an artificial neural network in patients undergoing radical cystectomy for bladder cancer: a comparison with logistic regression analysis. BJU Int. 2007;99(5):1007-12.

33. Batuello JT, Gamito EJ, Crawford ED, Han M, Partin AW, McLeod DG, et al. Artificial neural network model for the assessment of lymph node spread in patients with clinically localized prostate cancer. Urology. 2001;57(3):481-5.

34.Bollschweiler EH, Monig SP, Hensler K, Baldus SE, Maruyama K, Holscher AH. Artificial neural network for prediction of lymph node metastases in gastric cancer: a phase II diagnostic study. Ann Surg Oncol. 2004;11(5):506-11. 
35.Chi CL, Street WN, Wolberg WH. Application of artificial neural network-based survival analysis on two breast cancer datasets. AMIA Annu Symp Proc. 2007:130-4.

36.Crawford ED. Use of algorithms as determinants for individual patient decision making: national comprehensive cancer network versus artificial neural networks. Urology. 2003;62(6 Suppl 1):13-9.

37.Djavan B, Remzi M, Zlotta A, Seitz C, Snow P, Marberger M. Novel artificial neural network for early detection of prostate cancer. J Clin Oncol. 2002;20(4):921-9.

38.Floyd CE, Jr., Lo JY, Yun AJ, Sullivan DC, Kornguth PJ. Prediction of breast cancer malignancy using an artificial neural network. Cancer. 1994;74(11):2944-8.

39.Peng JH, Fang YJ, Li CX, Ou QJ, Jiang W, Lu SX, et al. A scoring system based on artificial neural network for predicting 10-year survival in stage II A colon cancer patients after radical surgery. Oncotarget. 2016;7(16):22939-47.

40.Shi HY, Lee KT, Lee HH, Ho WH, Sun DP, Wang JJ, et al. Comparison of artificial neural network and logistic regression models for predicting inhospital mortality after primary liver cancer surgery. PLoS One. 2012;7(4):e35781.

41.Shi HY, Tsai JT, Chen YM, Culbertson R, Chang HT, Hou MF. Predicting two-year quality of life after breast cancer surgery using artificial neural network and linear regression models. Breast Cancer Res Treat. 2012;135(1):221-9.

42.Gerlee P, Anderson AR. An evolutionary hybrid cellular automaton model of solid tumour growth. J Theor Biol. 2007;246(4):583-603.

43.Gerlee P, Anderson AR. A hybrid cellular automaton model of clonal evolution in cancer: the emergence of the glycolytic phenotype. J Theor Biol. 2008;250(4):705-22.

44.Gerlee P, Anderson AR. Evolution of cell motility in an individual-based model of tumour growth. J Theor Biol. 2009;259(1):67-83.

45.Gerlee P, Anderson AR. Modelling evolutionary cell behaviour using neural networks: application to tumour growth. Biosystems. 2009;95(2):166-74.

46.Gerlee P, Anderson AR. Diffusion-limited tumour growth: simulations and analysis. Math Biosci Eng. 2010;7(2):385-400.

47.Kim E, Kim JY, Smith MA, Haura EB, Anderson ARA. Cell signaling heterogeneity is modulated by both cell-intrinsic and -extrinsic mechanisms: An integrated approach to understanding targeted therapy. PLoS Biol. 2018;16(3):e2002930. 